\title{
Onset of Departure from Linearized Hydrodynamic Behavior in Argon Gas Studied with Neutron Brillouin Scattering
}

\author{
U. Bafile, (1), (a) P. Verkerk, ${ }^{(2)}$ F. Barocchi, (1) L. A. de Graaf, ${ }^{(2)}$ J.-B. Suck, ${ }^{(3)}$ and H. Mutka ${ }^{(4)}$ \\ ${ }^{(1)}$ Dipartimento di Fisica, Università di Firenze, 50125 Firenze, Italy \\ ${ }^{(2)}$ Interfacultair Reactor Instituut, Technische Universiteit Delft, 2629 JB Delft, The Netherlands \\ ${ }^{(3)}$ Institut für Nukleare Festkörperphysik, Kernforschungszentrum Karlsruhe, Karlsruhe, Federal Republic of Germany \\ ${ }^{(4)}$ Institut Laue-Langevin, 38042 Grenoble CEDEX, France
}

(Received 6 August 1990)

\begin{abstract}
We have investigated the dynamic structure factor of room-temperature ${ }^{36} \mathrm{Ar}$ at two densities $(0.25$ and 0.63 times the critical) and at very low momentum transfer $\left(0.3<\kappa<1.3 \mathrm{~nm}^{-1}\right)$. Linearized hydrodynamics agrees with the experimental data only at the higher density and $\kappa<1 \mathrm{~nm}^{-1}$. However, the description in terms of three extended hydrodynamic modes is fully satisfactory at both densities over the whole $\kappa$ range. We provide the first experimental evidence that the concept of extended hydrodynamic modes is valid for the whole range from hydrodynamics up into the kinetic regime.
\end{abstract}

PACS numbers: $61.20 . \mathrm{Lc}, 51.10 .+\mathrm{y}, 61.12 . \mathrm{Ex}$

The main topic of the physics of fluids is the development of a consistent framework in which macroscopic and microscopic properties are understood over the whole range of thermodynamic states. As far as dynamical properties are concerned, in order to fill the gap of knowledge between the two well-understood limiting cases of dilute gases and dense fluids in the hydrodynamic regime, theories have been developed by the generalization of the hydrodynamic approach ${ }^{1}$ and by the application of kinetic theory to dense fluids. ${ }^{2}$ Also, molecular-dynamics computer simulations have been performed for hard-sphere ${ }^{3}$ and Lennard-Jones systems ${ }^{4}$ in a wide region of the $(\kappa, \omega)$ space at intermediate and high densities. Here $\kappa$ and $\omega$ are the magnitude of the wave vector and the frequency of the dynamical processes in the fluid. The lowest $\kappa$ value obtained in these simulations was of the order of 1 or $2 \mathrm{~nm}^{-1}$.

In order to obtain experimental information on the transition between the two limiting dynamical regimes, the most interesting region for measurements of the $(\kappa, \omega)$ spectra of fluctuations in fluids at intermediate densities is for $0.3<\kappa l_{0}<3$, where $l_{0}$ is the mean free path of a corresponding hard-sphere system. Spectra connected to density fluctuations can be measured in this range of $\kappa$ only by means of thermal-neutron coherent scattering, where $\hbar \kappa$ and $\hbar \omega$ are identified with the momentum and energy transfer of the scattering process. Coherent neutron scattering at its low- $\kappa$ limit (i.e., neutron Brillouin scattering) probes density fluctuations on virtually the same spatial scale as Brillouin light scattering. In particular, in both cases a Rayleigh-Brillouin triplet should be observed in the hydrodynamic regime. ${ }^{1}$

The dynamic structure factor $S(\kappa, \omega)$ was measured in the past, by means of neutron Brillouin scattering, in two experiments in $\mathrm{Ar}$ (Ref. 5) and in $\mathrm{Ne}$ (Ref. 6) at various temperatures and densities above the critical one. However, the difficulties in performing such experiments are considerable and are related to the difficulty of measuring the scattering cross section at low enough $\kappa$ with sufficient resolution and intensity with an incident neutron speed significantly larger than the velocity of propagation of the excitation in the fluid.

Recently, the time-of-flight spectrometer IN5 at the Institut Laue-Langevin (ILL) in Grenoble has been modified in order to allow for precise small-angle measurements of $S(\kappa, \omega)$ in a region of $\kappa$ which almost completely covers the required transition region for gases at intermediate and high density, and Egelstaff et al. have performed a first experiment in nitrogen ${ }^{7}$ in which pronounced Brillouin peaks are visible. Later, the performance of the instrument was considerably improved, mainly in terms of reduction of background noise, and more accurate data have therefore been obtained in a number of new experiments on $\mathrm{N}_{2}, \mathrm{Kr}$, and ${ }^{36} \mathrm{Ar}$.

Here we report the results of an experiment, performed with the improved configuration of IN5, for the measurement of $S(\kappa, \omega)$ at small angles in roomtemperature ${ }^{36} \mathrm{Ar}$ at pressures of 8 and $20 \mathrm{MPa}$ corresponding to densities of 2.00 and 5.04 atoms nm ${ }^{-3}$, respectively, and to $\kappa l_{0}$ varying from 0.4 to 1.3 and from 0.15 to 0.5 , respectively. Some relevant thermodynamic properties of ${ }^{36} \mathrm{Ar}$ are summarized in Table I.

In order to optimize the scattering fraction, two different sample containers were used with single-crystal sapphire windows for the incident beam and for the scattered neutrons, which ensured a very low containerscattering signal. The residual background was mainly due to the scattering from natural argon in the $3.8-\mathrm{m}$ flightpath of IN5.

The small-angle time-of-flight detector consisted of an array of $64 \times 64$ elements of $1 \times 1 \mathrm{~cm}^{2}$ coded into concentric rings around the transmitted neutron beam, and was placed $3.8 \mathrm{~m}$ from the sample with the center of the detector shifted $15 \mathrm{~cm}$ away from the transmitted beam position in order to increase the scattering-angle range at one side of the scattering plane.

Incident neutrons with a wavelength of $0.6 \mathrm{~nm}$ were selected and the overall energy resolution at zero energy 
TABLE I. Thermophysical properties of ${ }^{36} \mathrm{Ar}$ at the thermodynamic states of the experiment. $n, \gamma, a, c_{s}, \eta_{s}$, and $S(0)$ are obtained from Ref. 14. $\Gamma$ is calculated with $\eta_{b} / \eta_{s}=0.05$ and 0.20 for the two states, respectively. The speed of incident neutrons was $0.659 \mathrm{~nm} \mathrm{ps}^{-1}$.

\begin{tabular}{|c|c|c|}
\hline Pressure $p\left(10^{5} \mathrm{~Pa}\right)$ & $80.35 \pm 0.05$ & $200.85 \pm 0.05$ \\
\hline Temperature $T(\mathrm{~K})$ & $301.5 \pm 1.0$ & $301.5 \pm 1.0$ \\
\hline Density $n\left(\mathrm{~nm}^{-3}\right)$ & 2.00 & 5.04 \\
\hline $\begin{array}{l}\text { Specific-heat } \\
\text { ratio } \gamma=C_{p} / C_{r}\end{array}$ & 1.905 & 2.211 \\
\hline $\begin{array}{l}\text { Thermal diffusivity } a \\
\left(\mathrm{~nm}^{2} \mathrm{ps}^{-1}\right)\end{array}$ & 0.270 & 0.117 \\
\hline $\begin{array}{l}\text { Adiabatic speed } \\
\text { of sound } c_{S}\left(\mathrm{~nm} \mathrm{ps}^{-1}\right)\end{array}$ & 0.353 & 0.393 \\
\hline $\begin{array}{l}\text { Shear viscosity } \eta_{s} \\
\left(10^{6} \mathrm{~kg} \mathrm{~m}^{-1} \mathrm{~s}^{-1}\right)\end{array}$ & 23.6 & 28.8 \\
\hline $\begin{array}{l}\text { Sound damping } \\
\text { factor } \Gamma\left(\mathrm{nm}^{2} \mathrm{ps}^{-1}\right)\end{array}$ & 0.254 & 0.134 \\
\hline$S(0)$ & 1.066 & 0.997 \\
\hline
\end{tabular}

transfer was $70 \mu \mathrm{eV}$ (measured with vanadium). The scattered intensity was measured at 42 scattering angles between $1.3^{\circ}$ and $7.2^{\circ}$ with a wave-vector transfer for elastic scattering $0.25<\kappa<1.30 \mathrm{~nm}^{-1}$.

The data have been analyzed by means of a welltested set of computer programs ${ }^{8}$ including statistical consistency checks, background and container-scattering subtraction, and correction for detector efficiency, resolution, ${ }^{9}$ multiple scattering, frame overlap, and selfshielding attenuation. The corrected scattered intensity is transformed into a symmetrized dynamic structure factor interpolated at a rectangular grid in the $(\kappa, \omega)$ plane. The data have been normalized to absolute units by means of an incoherent elastic-scattering measurement from a vanadium sample, which also served as a resolution measurement. It turned out to be difficult to achieve a very accurate normalization. Therefore, in the comparison with the theory, either the measured $S(\kappa)$ [obtained by integration of the experimental $S(\kappa, \omega)$ ] is used or $S(\kappa)$ has been obtained as a fit parameter.

For $\kappa<0.6 \mathrm{~nm}^{-1}$ at the higher density and for $\kappa<0.4 \mathrm{~nm}^{-1}$ at the lower density the width of $S(\kappa, \omega)$ is too small for the applied method of resolution correction $^{9}$ to be satisfactory. Outside this $\kappa$ range the correction was satisfactory, which was checked by folding one of the models [Eqs. (2) and (3)] with the experimental resolution function and comparing it with the uncorrected data.

Linearized hydrodynamics gives for the dynamic structure factor ${ }^{10}$

$$
S(\kappa, \omega)=\frac{S(\kappa)}{\pi} \operatorname{Re}\left(\frac{B(i \omega)}{M(i \omega)}\right),
$$

with

$$
B(z)=z^{2}+(\gamma a+v) \kappa^{2} z+\gamma a v \kappa^{4}+\frac{\gamma-1}{\gamma} c_{S}^{2} \kappa^{2},
$$

and

$M(z)=z^{3}+(\gamma a+v) \kappa^{2} z^{2}+\left(\gamma a v \kappa^{4}+c_{S}^{2} \kappa^{2}\right) z+c_{S}^{2} a \kappa^{4}$,

where $a=\lambda / n C_{p}$ is the thermal diffusivity, $\lambda$ the thermal conductivity, $n$ the number density, $C_{p}$ the specific heat at constant pressure, $v=\left(\eta_{b}+\frac{4}{3} \eta_{s}\right) / m n$ the kinematic longitudinal viscosity, $\eta_{s}$ the shear viscosity, $\eta_{b}$ the bulk viscosity, $m$ the atomic mass, $c_{S}$ the adiabatic speed of sound, and $\gamma=C_{p} / C_{v}$, the specific-heat ratio.

Equation ( $1 \mathrm{a})$ is equivalent to a sum of three Lorentzians:

$$
S(\kappa, \omega)=\frac{S(\kappa)}{\pi} \operatorname{Re}\left(\sum_{j=-1}^{1} \frac{A_{j}}{i \omega-z_{j}}\right),
$$

with $z_{j}$ the three complex solutions of $M(z)=0$, and $\sum A_{j}=1$. The central line $j=0$ ( $A_{0}$ and $z_{0}$ are real) represents a nonpropagating mode with $z_{H}=-z_{0}$ the damping coefficient. The two lines with $j= \pm 1(A \pm 1$ and $z \pm 1$ are complex-conjugated pairs) represent propagating modes with frequency $\omega_{S}=\operatorname{Im}(z-1)$ and damping coefficient $z_{S}=-\operatorname{Re}(z+1)$. The relations between the parameters in Eqs. (1a) and (1b) are given in Ref. 11. Explicitly,

$$
\begin{aligned}
S(\kappa, \omega)=\frac{S(\kappa)}{\pi}( & A_{0} \frac{z_{H}}{\omega^{2}+z_{H}^{2}}+A_{S} \frac{z_{S}+\left(\omega+\omega_{S}\right)^{b}}{\left(\omega+\omega_{S}\right)^{2}+z_{S}^{2}} \\
& \left.+A_{S} \frac{z_{S}-\left(\omega-\omega_{S}\right)^{b}}{\left(\omega-\omega_{S}\right)^{2}+z_{S}^{2}}\right)
\end{aligned}
$$

with $A_{S}=\operatorname{Re}\left(A_{+1}\right)$ and $b=\left[\left(A_{0} / 2 A_{S}\right) z_{H}+z_{S}\right] / \omega_{S}$, which gives the asymptotic behavior $S(\kappa, \omega)=O\left(1 / \omega^{4}\right)$ for large $\omega$ and ensures that the second frequency moment of $S(\kappa, \omega)$ is finite.

If $\gamma a \kappa^{2}$ and $v \kappa^{2}$ are small compared to $c_{S} \kappa$ [cf. Eq. (1a)], Eq. (2) reduces to the well-known RayleighBrillouin triplet ${ }^{1}$ with

$$
\begin{aligned}
& A_{0}=(\gamma-1) / \gamma, \quad A_{S}=1 / 2 \gamma, \quad \omega_{S}=c_{S} \kappa, \\
& z_{H}=a \kappa^{2}, \quad z_{S}=\Gamma \kappa^{2},
\end{aligned}
$$

where $\Gamma=\frac{1}{2}[v+(\gamma-1) a]$ is the sound damping factor.

We compare linearized hydrodynamics without the approximations of Eq. (3) with our experimental data. In Figs. 1 and 2 we display the measured $S(\kappa, \omega)$ together with Eq. (1) using the values reported in Table I for the thermophysical properties of ${ }^{36} \mathrm{Ar}$, the experimental integrated intensity for $S(k)$, and assuming $\eta_{b} / \eta_{s}=0.20$ and 0.05 for the higher and lower density, respectively, as given by the Enskog theory for hard spheres. ${ }^{12}$ This choice is based on the observation that $\eta_{b}$ can be obtained (rather inaccurately) from a fit of Eq. (1) to the data at $20 \mathrm{MPa}$ and appears to be consistent with the Enskog value. At $8 \mathrm{MPa}$ no acceptable fit is obtained, but the sum of squares tends to decrease with decreasing $\eta_{b}$. We note from Eq. (2) that the intensity increases in the central peak and decreases in the side line with in- 


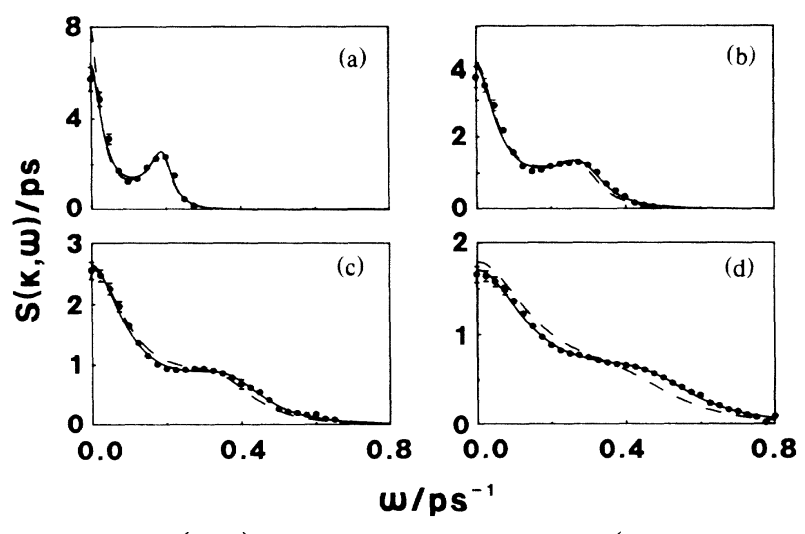

FIG. 1. $S(\kappa, \omega)$ at $20 \mathrm{MPa}$ : experiment (dots with error bars), linearized hydrodynamics (dashed line), linearized hydrodynamics with fitted parameters (solid line). (a) $\kappa=0.50$ $\mathrm{nm}^{-1}$; (b) $\kappa=0.75 \mathrm{~nm}^{-1}$; (c) $\kappa=1.00 \mathrm{~nm}^{-1}$; (d) $\kappa=1.25$ $\mathrm{nm}^{-1}$.

creasing $\eta_{b}$, but the overall shape of the measured spectrum is reproduced only for the lowest $\kappa$ values at the higher density (see Figs. 1 and 2).

Next, we apply the concept of extended hydrodynamics, ${ }^{2}$ i.e., Eqs. (2) and (3), with $a, \Gamma$, the intensity ratio of the side line to the central one $r=A_{S} / A_{0}$, and $c_{S}$ all $\kappa$ dependent. $S(\kappa), z_{H}(\kappa), z_{S}(\kappa), r(\kappa)$, and $\omega_{S}(\kappa)$ were determined from a least-squares fit of Eq. (2) to the neutron data. Since at both densities $\omega_{S}$ shows the linear behavior of Eq. (3), a new fitting has been performed with only the first four free parameters and $\omega_{S}=c_{S} \kappa$, thus obtaining a better determination of the other parameters.

The fitted $S(\kappa, \omega)$, shown in Figs. 1 and 2, gives an ex-

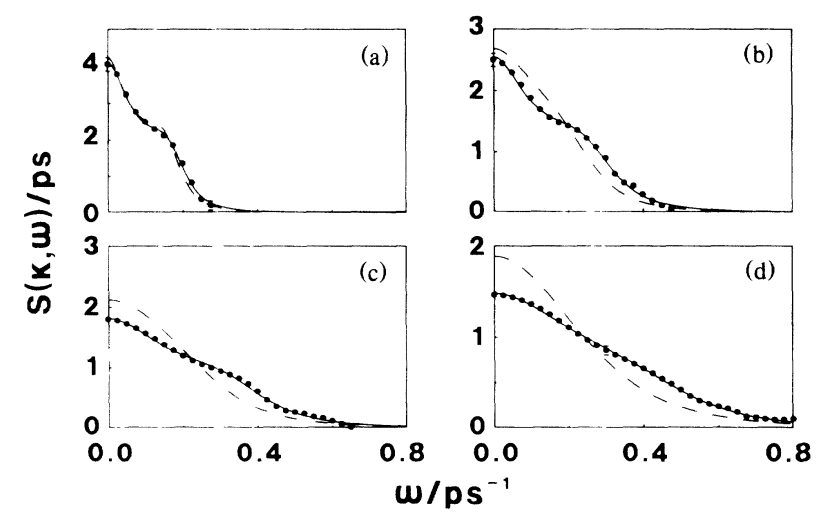

FIG. 2. $S(\kappa, \omega)$ at $8 \mathrm{MPa}$. Symbols as in Fig. 1. (a) $\kappa=0.50 \mathrm{~nm}^{-1}$; (b) $\kappa=0.75 \mathrm{~nm}^{-1}$; (c) $\kappa=1.00 \mathrm{~nm}^{-1}$; (d) $\kappa=1.25 \mathrm{~nm}^{-1}$.

cellent agreement with the data at all $\kappa$ 's and both densities. In order to get some insight into the deviation from linearized hydrodynamics, in Fig. 3 we compare the $\kappa$ dependence of the fitted parameters with the prediction of Eq. (1), where again the Enskog value of $\eta_{b} / \eta_{s}$ is used [for completeness we also display Eqs. (3)]. It should be noted that the mode-coupling theory ${ }^{13}$ predicts for the parameters $z_{H}, z_{S}$, and $\omega_{S}$ an asymptotic behavior towards the $\kappa \rightarrow 0$ limit where the first correction to the hydrodynamic expressions of Eq. (3) is proportional to $\kappa^{5 / 2}$. For $\kappa=1.5 \mathrm{~nm}^{-1}$ the relative corrections introduced by this term for $z_{H}, z_{S}$, and $\omega_{S}$ are $0.3 \%, 1.4 \%$, and $1.5 \%$ for $p=8 \mathrm{MPa}$, respectively, and $0.6 \%, 4.0 \%$, and $2.0 \%$ for $p=20 \mathrm{MPa}$, respectively, and are too small
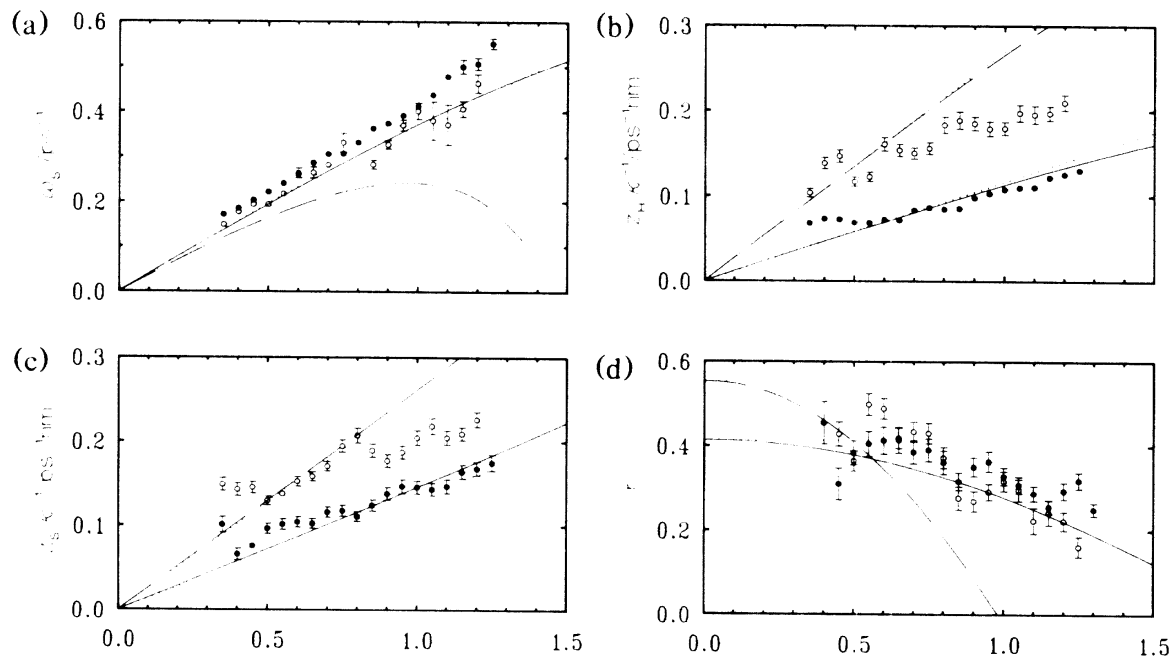

FIG. 3. Parameters of the linearized hydrodynamic model: experimental data (dots with error bars at $20 \mathrm{MPa}$, open circles with error bars at $8 \mathrm{MPa}$ ) and theory (solid line at $20 \mathrm{MPa}$ and dashed line at $8 \mathrm{MPa}$ ). The dotted lines indicate Eq. (3) for the two densities. (a) Peak position $\omega_{S}$ of the Brillouin line; (b) half width at half maximum $z_{H}$ of the Rayleigh line divided by $\kappa$; (c) half width at half maximum $z_{S}$ of the Brillouin line divided by $\kappa$; (d) ratio $r$ of intensities of Brillouin to Rayleigh line. 
to be possibly detected from the experimental data.

We have measured the dynamic structure factor in argon at room temperature at densities of 0.25 and 0.6 times the critical density. The measurements cover $0.15<\kappa l_{0}<1.3$, where $\kappa$ is the magnitude of the wave vector of the density fluctuations and $l_{0}$ is the mean free path of an equivalent hard-sphere gas. We observe within this range a gradual transition of $S(\kappa, \omega)$ from a triplet to a single central peak. The comparison of the fit results with the theory shows that at the higher density the $\kappa$ dependence of the fitted parameters agrees with the prediction of linearized hydrodynamics, with slight deviations for $\omega_{S}, z_{H}$, and $r$ at $\kappa>0.9 \mathrm{~nm}^{-1}\left(\kappa l_{0}\right.$ $>0.35)$. At the lower density, deviations appear at $\kappa>0.9 \mathrm{~nm}^{-1}\left(\kappa l_{0}>0.9\right)$ for $z_{S}$ and at $\kappa>0.6 \mathrm{~nm}^{-1}$ $\left(\kappa l_{0}>0.6\right)$ for the other parameters. In particular, $z_{H}$ seems to tend to a linear dependence on $\kappa$ which is the typical kinetic behavior of a low-density system. We believe that this is the first clear sign of the onset of the transition between the hydrodynamic and kinetic regimes in gases at intermediate densities.

Over the whole range of $\kappa l_{0}$ studied here the description with a sum of three Lorentzians (i.e., extended hydrodynamic modes) is accurate within the experimental errors. It should be noted, that outside the hydrodynamic region the dynamics in liquid argon, ${ }^{15}$ liquid neon, ${ }^{11}$ hard-sphere fluids, ${ }^{3}$ and Lennard-Jones fluids ${ }^{4}$ can all be described satisfactorily in terms of three extended hydrodynamic modes. We provide the first experimental evidence that the three hydrodynamic modes go over in a continuous way into three extended hydrodynamic modes. Thus, the present data support the analysis in terms of extended hydrodynamic modes of neutron data and computer simulations at larger $\kappa$ values. ${ }^{3,4,11,15}$

U.B. acknowledges the award of a NATO-Consiglio Nazionale delle Ricerche fellowship for his stay at the IRI, Delft. P.V. acknowledges the financial support of NWO (Nederlandse Organisatie voor Wetenschappelijk Onderzoek) and CNRS (Centre National da la Recherche Scientifique). We thank P. v. d. Ende, V.-O. de Haan, and Mr. Milesi and his staff of ILL for their skillful assistance. We are indebted to G. Kearley and S.
Jenkins (ILL) for the successful modification of IN5. We thank P. A. Egelstaff, J. P. A. Youden, and I. M. de Schepper for stimulating discussions.

(a) Permanent address: Istituto di Elettronica Quantistica,
Consiglio Nazionale delle Ricerche, 50127 Firenze, Italy.
'J. P. Boon and S. Yip, Molecular Hydrodynamics

${ }^{2}$ I. M. de Schepper and E. G. D. Cohen, Phys. Rev. A 22, 287 (1980); J. Stat. Phys. 27, 223 (1982); B. Kamgar-Parsi, E. G. D. Cohen, and I. M. de Schepper, Phys. Rev. A 35, 4781 (1987).

${ }^{3}$ W. E. Alley and B. J. Alder, Phys. Rev. A 27, 3158 (1983); W. E. Alley, B. J. Alder, and S. Yip, Phys. Rev. A 27, 3174 (1983); C. Bruin, J. P. J. Michels, J. C. van Rijs, L. A. de Graaf, and I. M. de Schepper, Phys. Lett. 110A, 40 (1985).

${ }^{4}$ I. M. de Schepper, J. C. van Rijs, A. A. van Well, P. Verkerk, and L. A. de Graaf, Phys. Rev. A 29, 1602 (1984).

${ }^{5}$ T. A. Postol and C. A. Pelizzari, Phys. Rev. A 18, 2321 (1978)

${ }^{6}$ H. G. Bell, A. Kollmar, B. Alefeld, and T. Springer, Phys. Lett. 45A, 479 (1973); H. Bell, H. Moeller-Wenghoffer, A. Kollmar, R. Stockmeyer, T. Springer, and H. Stiller, Phys. Rev. A 11, 316 (1975).

${ }^{7}$ P. A. Egelstaff, G. Kearley, J.-B. Suck, and J. P. A. Youden, Europhys. Lett. 10, 37 (1989).

${ }^{8}$ P. Verkerk and A. A. van Well, Nucl. Instrum. Methods Phys. Res., Sect. A 228, 438 (1985).

${ }^{9}$ P. Verkerk, Comput. Phys. Commun. 25, 325 (1982).

${ }^{10}$ R. D. Mountain, Rev. Mod. Phys. 38, 205 (1966).

${ }^{11}$ A. A. van Well and L. A. de Graaf, Phys. Rev. A 32, 2396 (1985).

${ }^{12} \mathrm{~S}$. Chapman and T. G. Cowling, The Mathematical Theory of Nonuniform Gases (Cambridge Univ. Press, Cambridge, 1970).

${ }^{13}$ M. H. Ernst and J. R. Dorfman, J. Stat. Phys. 12, 311 (1975).

${ }^{14}$ V. A. Rabinovich, A. A. Vasserman, V. I. Nedostup, and L. S. Veksler, Thermophysical Properties of Neon, Argon, Krypton and Xenon (Hemisphere, Washington, 1988).

${ }^{15}$ I. M. de Schepper, P. Verkerk, A. A. van Well, and L. A. de Graaf, Phys. Rev. Lett. 50, 974 (1983); P. Verkerk and A. A. van Well, Physica (Amsterdam) 136B, 168 (1986). 